Comment. Math. Helv. 79 (2004) 502-515

0010-2571/04/030502-14

DOI 10.1007/s00014-004-0804-2 (c) 2004 Birkhäuser Verlag, Basel

Commentarii Mathematici Helvetici

\title{
On the Gabai-Eliashberg-Thurston Theorem
}

\author{
Ko Honda, William H. Kazez and Gordana Matić
}

\begin{abstract}
We present a new, completely three-dimensional proof of the fact, due to the combined work of Gabai and Eliashberg-Thurston, that every closed, oriented, connected, irreducible 3-manifold with nonzero second homology carries a universally tight contact structure.
\end{abstract}

Mathematics Subject Classification (2000). Primary 57M50; Secondary 53C15.

Keywords. Tight, contact structure.

\section{Introduction}

A contact structure on a 3 -manifold $M$ is a completely nonintegrable field $\xi$ of tangent 2-planes on $M$. It is locally defined as the kernel of a smooth 1-form $\alpha$ such that $\alpha \wedge d \alpha$ is nowhere vanishing. A contact structure $\xi$ on $M$ is called tight if there is no embedded disc $D^{2} \subset M$ such that for all points in $\partial D$, the tangent space to $D$ is equal to $\xi$. If there is such an overtwisted disc $D$, the contact structure is called overtwisted. Bennequin [2] showed that the standard contact structure on $\mathbb{R}^{3}$ is tight. Eliashberg [5] showed that the classification of overtwisted contact structures up to isotopy on an arbitrary closed 3-manifold coincides with their homotopy classification as 2-plane fields. This gives a complete answer to the question of existence and uniqueness for overtwisted structures.

Until recently, to decide if a contact structure is tight or to construct tight contact structures, one of two techniques was used. Either the contact structure was shown to be semi-fillable (i.e., a component of the convex boundary of a symplectic manifold), hence tight by Eliashberg and Gromov [7], [15], or it had a tight cover. One rich source of fillable examples is surgery on Legendrian links [6], [14]. The other is a theorem of Thurston and Eliashberg [9] that a taut foliation can be perturbed to obtain a semi-fillable contact structure. This, coupled with the work of Gabai [10] on the existence of taut foliations, gives the most general existence theorem to date.

KH supported by NSF grant DMS-0072853; GM supported by NSF grant DMS-0072853; WHK supported by NSF grant DMS-0073029. 
Theorem 0.1 (Gabai-Eliashberg-Thurston). Let $M$ be a closed, oriented, connected, irreducible 3-manifold with $H_{2}(M, \mathbb{Z}) \neq 0$. Then $M$ carries a universally tight contact structure.

In [20], the authors proved the following variant of the above theorem for manifolds with boundary:

Theorem 0.2. Let $(M, \gamma)$ be an oriented, compact, connected, irreducible, sutured 3-manifold which has nonempty boundary, is taut, and has annular sutures. Then $(M, \gamma)$ carries a universally tight contact structure.

Our approach to contact topology is built upon the seminal work of Giroux [11]. He identified and developed one of the crucial tools in the subject, convex surfaces, and described how a family of closed curves $\Gamma_{\Sigma}$ on the surface $\Sigma$, the dividing set of $\Sigma$, determines the contact structure in the neighborhood of the convex surface. This notion was extended to surfaces with Legendrian boundary by Kanda [22], [23]. In [13] Giroux described what happens to a convex surface during an isotopy by something like a movie of the changing dividing set. Honda [16] defined the notion of a bypass to capture the most basic change of the dividing set under an isotopy. In [19, 20], we defined the notion of a convex decomposition that is the contact topology analogue of Haken decompositions and sutured manifold decompositions. This has allowed us to both classify and construct tight contact structures. One can think of these methods as cut-and-paste contact topology: we analyze and build tight contact structures by decomposing them and/or gluing them back together along convex surfaces.

Our interest in reproving Theorem 0.1 is twofold. First, if the starting point is a sutured manifold decomposition and the goal is to build a universally tight contact structure, it should not be necessary (indeed it is not) to construct a taut foliation à la Gabai [10], perturb it into a contact structure, and argue using symplectic filling techniques that the resulting contact structure is universally tight (this was the path taken by Eliashberg-Thurston in [9]). Our other motivation is to use these theorems as guidelines in the development of a cut-and-paste theory of contact topology. This theory contrasts with foliation theory right from the start. Given a tight contact structure, it is very easy to produce convex decompositions of the space (see [20]). On the other hand, given two pieces of a manifold, each with a universally tight contact structure, it is surprisingly difficult to find gluing theorems which allow one to conclude that the contact structure on their union is universally tight. In the theory of taut foliations, the relative difficulty levels of the two appear to be switched.

We have used the search for cut-and-paste proofs of the Gabai-EliashbergThurston theorem as a method for finding new gluing theorems. Theorem 0.2 was proved using gluing techniques (pioneered by Colin $[3,4]$ ). The key gluing theorem used in the proof was: 
Theorem 0.3 (Colin [4]). Let $(M, \xi)$ be an oriented, compact, connected, irreducible, contact 3-manifold and $S \subset M$ an incompressible convex surface with nonempty Legendrian boundary and $\partial$-parallel dividing set $\Gamma_{S}$. If $\left(M \backslash S,\left.\xi\right|_{M \backslash S}\right)$ is universally tight, then $(M, \xi)$ is universally tight.

The condition $\partial S \neq \emptyset$ is important in the proof of Theorem 0.3 . Therefore, Theorem 0.3 is not applicable when $M$ is a closed 3 -manifold and the first cut in the sutured manifold decomposition is along a closed surface.

There is also a gluing theorem along incompressible tori, due to Colin [4]. In [20], we rephrased and gave a slightly different proof of this result, and presented a foliation-theory-free proof of the existence theorem for tight contact structures in the case of a closed, irreducible, toroidal manifold (without the assumption $\left.H_{2}(M, \mathbb{Z}) \neq 0\right)$.

The main Gluing Theorem of this paper is in many ways a simultaneous generalization of the above two gluing theorems - along tori and along surfaces with boundary. For the purposes of this paper, it suffices to prove a gluing result for atoroidal manifolds, along closed convex surfaces $\Sigma$ of genus $g>1$ that satisfy the extremal condition

$$
\langle e(\xi), \Sigma\rangle= \pm(2 g-2),
$$

where the left-hand side is the Euler class of $\xi$ evaluated on $\Sigma$. (Note that the condition is trivially satisfied for genus one surfaces.) Tight contact structures $\xi$ on $M$ satisfying this condition are said to be extremal along $\Sigma$, since

$$
-(2 g-2) \leq\langle e(\xi), \Sigma\rangle \leq 2 g-2,
$$

by the Bennequin inequality [2, 8]. With the understanding that the necessary terminology will be explained in Section 1, the precise statement of the Gluing Theorem is as follows:

Theorem 0.4 (Gluing Theorem). Let $(M, \xi)$ be an oriented, compact, irreducible, atoroidal contact manifold which is extremal along a closed, convex, incompressible surface $\Sigma$, and let $\Sigma^{0}$ and $\Sigma^{1}$ be the boundary components of $M \backslash \Sigma$ corresponding to $\Sigma$. Suppose that, for each $i=0,1, \Sigma^{i}$ is fully straddled in $M \backslash \Sigma$ with a straddling set $\mathbb{S}^{i}$, and the dividing curves of $\Sigma^{i}$ are straddled if and only if they are unstraddled in $\Sigma^{1-i}$. Then $(M, \xi)$ is universally tight if and only if $\left(M \backslash \Sigma,\left.\xi\right|_{M \backslash \Sigma}\right)$ is universally tight.

The two key ingredients in the proof of Theorem 0.1 are the above Gluing Theorem (Theorem 0.4) along closed surfaces $\Sigma$ satisfying the extremal condition and a good understanding of universally tight contact structures on $\Sigma \times[0,1]$. One of the main results of [21] is Theorem 0.5 , which gives the classification of extremal tight contact structures on $\Sigma \times[0,1]$ in the case of two parallel dividing curves on each boundary component. This result, and its implications for covering spaces of $\Sigma \times[0,1]$, are enough to construct contact structures satisfying the hypotheses of the Gluing Theorem. 
Theorem 0.5. Let $\Sigma$ be a closed oriented surface of genus $g \geq 2$ and $M=\Sigma \times[0,1]$. Write $\Sigma_{i}=\Sigma \times\{i\}, i=0,1$. Suppose that $\Gamma_{\Sigma_{i}}$ is the union of a pair $\gamma_{i}, \gamma_{i}^{\prime}$ of parallel nonseparating curves which cobound an annulus $A_{i} \subset \Sigma_{i}$ and that $\Gamma_{\Sigma_{0}}$ is not isotopic to $\Gamma_{\Sigma_{1}}$. (Here the $\Sigma_{i}$ are oriented in the same way, and $A_{i}$ is the negative region $\left(\Sigma_{i}\right)_{-}$of $\Sigma_{i} \backslash \Gamma_{\Sigma_{i}}$.) Choose a characteristic foliation $\mathcal{F}$ on $\partial M$ which is adapted to $\Gamma_{\Sigma_{0}} \sqcup \Gamma_{\Sigma_{1}}$. Then there exist, up to isotopy rel boundary, exactly 4 tight contact structures which satisfy the boundary condition $\mathcal{F}$, and all of them are universally tight. Moreover, for each of the 4 tight contact structures, both $\Sigma_{0}$ and $\Sigma_{1}$ are fully straddled, and the 4 cases correspond to the 4 possible choices for the set of straddled curves $\Gamma(\mathbb{S})$, consisting of exactly one curve from each $\Gamma_{\Sigma_{i}}$.

Observe that Theorem 0.1 is an existence result, whose proof relies only on the existence portion of Theorem 0.5. Since the existence of the 4 universally tight contact structures is much easier to prove than the full classification theorem, the proof is given in the Appendix, where we derive the existence from Theorems 0.3 and 0.4 .

In Section 1, we introduce the notion of straddling and explain the basic properties. Section 2 contains a proof of the Gluing Theorem. In Section 3, Gabai's well-groomed sutured manifold decomposition theory is used to construct a universally tight contact structure on the cut-open manifold, and then the Gluing Theorem completes the proof of Theorem 0.1.

We adopt the following conventions:

(1) The ambient manifold $M$ is an oriented, compact 3-manifold.

(2) $\xi=$ positive contact structure which is co-oriented by a global 1-form.

(3) A convex surface $S$ is either closed or compact with Legendrian boundary.

(4) $\Gamma_{S}=$ dividing multicurve of a convex surface $S$.

(5) $\# \Gamma_{S}=$ number of connected components of $\Gamma_{S}$.

(6) $S \backslash \Gamma_{S}=S_{+} \cup S_{-}$, where $S_{+}$(resp. $S_{-}$) is the region where the normal orientation of $S$ is the same as (resp. opposite to) the normal orientation for $\xi$.

(7) $|\alpha \cap \beta|=$ geometric intersection number of two curves $\alpha$ and $\beta$.

Acknowledgements. The first author thanks the American Institute of Mathematics, Stanford University, and IHES for their hospitality. The authors also thank the referee for pointing out that this paper could be made self-contained by including a proof of the existence portion of Theorem 0.5.

\section{Straddling}

The following proposition will be used repeatedly throughout the paper.

Proposition 1.1 (Add = dig). Let $(M, \xi)$ be a tight contact 3-manifold with convex boundary, $\Sigma$ a component of $\partial M$, and $\gamma, \gamma^{\prime}$ a pair of parallel disjoint curves 
in $\Gamma_{\Sigma}$. Suppose there is a bypass $\mathcal{B}_{\alpha} \subset M$ attached along an arc $\alpha \subset \Sigma$ that starts on $\gamma^{\prime}$, crosses $\gamma$, and then ends on a third curve in $\Gamma_{\Sigma}-\left(\gamma \cup \gamma^{\prime}\right)$. Let $\beta$ be a Legendrian arc in $\Sigma$ that starts on $\gamma$, crosses $\gamma^{\prime}$, ends on a point of $\Gamma_{\Sigma}-\left(\gamma \cup \gamma^{\prime}\right)$, and does not intersect $\alpha$ or any other points of $\Gamma_{\Sigma}$. Then attaching a bypass $\mathcal{B}_{\beta}$ to $M$ along $\beta$ produces a manifold contact isomorphic to the manifold obtained by removing a convex neighborhood of $\mathcal{B}_{\alpha}$.

Proof. Let $B \subset \Sigma$ be a regular neighborhood of the union of $\alpha, \beta$, and the annulus in $\Sigma$ bounded by $\gamma$ and $\gamma^{\prime}$ - we assume $B$ is convex with Legendrian boundary. Let $V$ be a small neighborhood of the union of $B, \mathcal{B}_{\alpha}$, and $\mathcal{B}_{\beta}$. See Figure 1 . Topologically, $V$ is the product of an annulus and an interval, i.e., a solid torus. After the necessary edge-rounding, we see that the dividing set of $\partial V$ has two components, each of which intersects a compressing disk in a single point. There is a unique tight contact structure on $V$ with this boundary condition, as can be seen by splitting $V$ along the compressing disk and appealing to Eliashberg's uniqueness theorem on a ball [8]. It remains to verify that the contact structure on $V$ is indeed tight - for this we simply remark that an explicit model can be found inside the unique (product) tight contact structure on $V$. Since the contact structure on $V$ is a product, it follows that adding a bypass along $\beta$ is equivalent to removing a bypass along $\alpha$.
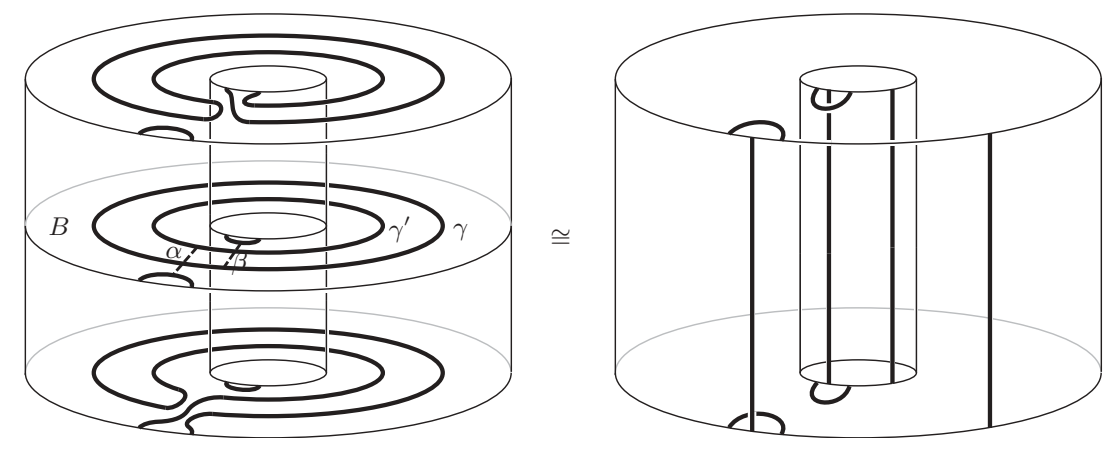

FIG. 1. Neighborhood of $B$

Let $(M, \xi)$ be a tight contact 3-manifold with convex boundary and let $\Sigma$ be a connected component of $\partial M$ of genus $g$ which satisfies the extremal condition $\langle e(\xi), \Sigma\rangle=-(2 g-2)$. It follows that $\Sigma_{-}$has zero Euler characteristic and is a disjoint union of annuli $A_{i}$, for $i=1, \ldots, n$. Denote $\partial A_{i}=\gamma_{i}^{0} \cup \gamma_{i}^{1}$, and call $\gamma_{i}^{0}$ and $\gamma_{i}^{1}$ a parallel pair of dividing curves.

We say a dividing curve $\gamma_{i}^{j}$ is straddled if there is a bypass in $M$ with an attaching arc $\alpha_{i}^{j}$ which starts on $\gamma_{i}^{1-j}$, crosses $\gamma_{i}^{j}$, and ends on (i) a point of $\Gamma_{\Sigma}-\left(\gamma_{i}^{0} \cup \gamma_{i}^{1}\right)$ or on (ii) $\gamma_{i}^{0}$ or $\gamma_{i}^{1}$, but only after first going around a nontrivial loop on $\Sigma$ which is not parallel to $\gamma_{i}^{j}$. Our bypasses may be degenerate, i.e., the 
two endpoints of the arc of attachment are allowed to coincide. The attaching arcs of these bypasses are called straddling arcs.

A closed convex surface $\Sigma$ of genus $g>1$ which is a connected component of $\partial M$ is fully straddled if the following hold:

(1) $\xi$ is extremal along $\Sigma$ and satisfies $\langle e(\xi), \Sigma\rangle=-(2 g-2)$.

(2) The components of $\Sigma_{-}$are pairwise nonparallel annuli.

(3) There is a collection $\mathbb{S}=\left\{\alpha_{i}\right\}$ of straddling arcs (called a straddling set) and a corresponding collection of bypasses $\mathbb{B}=\left\{\mathcal{B}_{\alpha_{i}}\right\}$ in $M$ such that:

(a) at least one curve in each parallel pair of $\Gamma_{\Sigma}$ is straddled by a bypass in $\mathbb{B}$,

(b) every curve in $\Gamma_{\Sigma}$ is straddled by at most one bypass in $\mathbb{B}$,

(c) if $i \neq j$, then either $\mathcal{B}_{\alpha_{i}}$ and $\mathcal{B}_{\alpha_{j}}$ are disjoint or intersect only at the endpoints of their corresponding arcs of attachment, and

(d) $\mathbb{S}$ is an essential family, i.e., $\Sigma_{+}-\cup_{i=1}^{n} \alpha_{i}$ has no disk components. (Equivalently, a thickening of $\Sigma_{-} \cup\left(\cup_{i=1}^{n} \alpha_{i}\right)$ is an incompressible subsurface of $\Sigma$.)

Remark 1.2. Condition $3(\mathrm{~d})$ is a sufficient condition to ensure that homotopically trivial dividing curves do not appear after all the bypasses of $\mathbb{B}$ are attached onto $\Sigma$.

The following two propositions describe the persistence of full straddlings under the operations of taking covers and attaching bypasses.

Proposition 1.3. Let $(M, \xi)$ be a tight contact manifold with convex boundary, and let $(\widetilde{M}, \widetilde{\xi})$ be a finite cover. If $\Sigma$ is a boundary component of $(M, \xi)$ which is fully straddled, then the preimage $\widetilde{\Sigma}$ of $\Sigma$ is fully straddled in $(\widetilde{M}, \widetilde{\xi})$.

Proof. Let $\mathbb{S}$ be the straddling set for $\Sigma$ and $\mathbb{B}$ the corresponding set of bypasses. Their preimages $\widetilde{\mathbb{S}}$ and $\widetilde{\mathbb{B}}$ satisfy all of the axioms necessary for $\widetilde{\Sigma}$ to be fully straddled, except that there may be several arcs in $\widetilde{\mathbb{S}}$ which straddle the same dividing curve of $\widetilde{\Sigma}$. Removing extra components of $\widetilde{\mathbb{S}}$ decreases the Euler characteristics of the complementary regions. Thus no disk components are produced, and $\widetilde{\Sigma}$ is fully straddled.

Proposition 1.4. Let $(M, \xi)$ be a tight contact manifold with convex boundary, $\Sigma$ a boundary component of $M$ which is fully straddled, and $\mathbb{S}$ a corresponding straddling set. Let $\alpha$ be an arc of $\mathbb{S}$ which straddles the dividing curve $\gamma$. Suppose there exists an arc $\alpha^{\prime} \subset \Sigma$ disjoint from $\alpha$ such that $\alpha^{\prime}$ starts on $\gamma$, crosses the dividing curve $\gamma^{\prime}$ parallel to $\gamma$, and ends on a point of $\Gamma_{\Sigma}-\left(\gamma \cup \gamma^{\prime}\right)$, while intersecting no other points of $\Gamma_{\Sigma}$. Then the manifold $\left(M^{\prime}, \xi^{\prime}\right)$, obtained by attaching a bypass along $\alpha^{\prime}$ to $(M, \xi)$, also is fully straddled along the boundary component $\Sigma^{\prime}$ corresponding to $\Sigma$. If $(M, \xi)$ is (universally) tight, then so is $\left(M^{\prime}, \xi^{\prime}\right)$. 
Proof. By Proposition 1.1, attaching a bypass along $\alpha^{\prime}$ to $(M, \xi)$ is equivalent to digging $\mathcal{B}_{\alpha}$ from $(M, \xi)$. Hence if $(M, \xi)$ is (universally) tight, then $\left(M^{\prime}, \xi^{\prime}\right)$ is also (universally) tight.

We now verify the full straddling. Observe that digging the bypass $\mathcal{B}_{\alpha}$ to obtain $\Sigma^{\prime}$ decreases $\# \Gamma_{\Sigma}$ by two. The new collection of straddling arcs $\mathbb{S}^{\prime}$ is defined to be $\mathbb{S}-\{\alpha\}$. To see that $\mathbb{S}^{\prime}$ is an essential family of arcs, it is necessary to relate the complementary region $\Omega^{\prime}=\Sigma_{+}^{\prime}-\left(\cup_{\beta \in \mathbb{S}^{\prime}} \beta\right)$ to the corresponding complementary region $\Omega=\Sigma_{+}-\left(\cup_{\beta \in \mathbb{S}} \beta\right)$. Now, it is not difficult to see that $\Omega^{\prime}$ is obtained from $\Omega$ by attaching a band. This can only decrease the Euler characteristic of the complementary regions. Hence $\mathbb{S}^{\prime}$ is an essential family.

Given a connected component $\Sigma$ of $\partial M$ which is fully straddled, define $\Gamma(\mathbb{S})$ to be the union of dividing curves of $\Sigma$ which are straddled by the (full) straddling set $\mathbb{S}$. In view of the following proposition, $\Gamma(\mathbb{S})$ is an invariant of the universally tight contact structure $\xi$ (under suitable conditions).

Proposition 1.5. Let $M$ be a compact, oriented, hyperbolic 3-manifold (i.e., $\operatorname{int}(M)$ admits a complete hyperbolic metric), $\xi$ be a universally tight contact structure on $M$, and $\Sigma$ be an incompressible, fully straddled component of $\partial M$. If $\gamma$ and $\gamma^{\prime}$ form a parallel pair of $\Gamma_{\Sigma}$ and $\gamma$ is straddled, then $\gamma^{\prime}$ is unstraddled, i.e., there is no bypass in $(M, \xi)$ which straddles $\gamma^{\prime}$.

Proof. We argue by contradiction. Let $\mathbb{S}$ be a full straddling set for $\Sigma, \mathbb{B}$ be the corresponding set of bypasses, and $\alpha$ be the arc in $\mathbb{S}$ which straddles $\gamma$. Suppose there exists a straddling arc $\beta$ for $\gamma^{\prime}$.

Claim. There is a finite cover $\pi:(\widetilde{M}, \widetilde{\xi}) \rightarrow(M, \xi)$ in which components $\widetilde{\alpha}$ and $\widetilde{\beta}$ of preimages of $\alpha$ and $\beta$ straddle a parallel pair of dividing curves $\widetilde{\gamma}$ and $\widetilde{\gamma}^{\prime}$, and are disjoint.

Proof of Claim. To prove the claim we use the following theorem of AllmanHamilton [1].

Theorem 1.6 (Abelian subgroup separability). Let $M$ be a hyperbolic 3-manifold. Then abelian subgroups $H$ of $\pi_{1}(M, *)$ are separable, i.e., for any $g \in \pi_{1}(M, *)-H$ there exists a finite index subgroup $K \supset H$ which does not contain $g$.

Let $\sigma$ be the core of the annulus $A$ bounded by $\gamma$ and $\gamma^{\prime}$. If $\alpha$ intersects only two dividing curves, let $\bar{\alpha}$ be a closed loop formed by the union of $\alpha$ and a subarc of $\sigma$. From the definition of a straddling arc, $\bar{\alpha}$ is not in the subgroup $\langle\sigma\rangle$ generated by $\sigma$. According to Theorem 1.6, after passing to a finite cover, we may assume that $\alpha$ intersects 3 distinct dividing curves. Similarly, we may assume that $\beta$ intersects 3 distinct dividing curves. Next, by applying Theorem 1.6 to the 
trivial subgroup $H=\{e\}$ (or by using residual finiteness), there exists a finite cover $\widetilde{M}$ whose fundamental group does not contain $\sigma^{k}$. Let $\widetilde{\alpha}$ be a component of the preimage of $\alpha$ which intersects a component $\widetilde{A}$ of the preimage of $A$ in an arc. If $k$ is large enough, there is a component $\widetilde{\beta}$ of the preimage of $\beta$ which straddles the other boundary component of $\widetilde{A}$ and is disjoint from $\widetilde{\alpha}$. This proves the claim.

Now, Proposition 1.1 shows that attaching a bypass along $\widetilde{\beta}$ from the exterior of $\widetilde{M}$ produces a contact structure isomorphic to the one obtained by digging out the bypass in $\widetilde{M}$ attached along $\widetilde{\alpha}$, and, in particular, this new contact structure must be universally tight. On the other hand, $\widetilde{\beta}$ is assumed to be the attaching arc for a bypass in $\widetilde{M}$, and it follows that attaching a bypass along $\widetilde{\beta}$ to the outside of $\widetilde{M}$ produces an overtwisted disk. This is a contradiction.

\section{Gluing along surfaces which are fully straddled}

Proof of Theorem 0.4. The proof that $(M, \xi)$ is tight also applies to finite covers of $(M, \xi)$, in light of Proposition 1.3. The atoroidal Haken hypothesis allows us to assume that $M$ is hyperbolic. As a consequence, $M$ has a residually finite fundamental group. Therefore, any overtwisted disk that exists in the universal cover also exists in some finite cover, and the proof below will also imply that $(M, \xi)$ is universally tight.

The general strategy for proving tightness is explained in detail in [20] and [18], so we will only provide a brief summary. Arguing by contradiction, we assume $(M, \xi)$ contains an overtwisted disk $D$. There exists a sequence $\Sigma=\Sigma_{0}, \Sigma_{1}, \ldots, \Sigma_{n}$ of isotopic surfaces, where each step is a single bypass attachment and $\Sigma_{n}$ is disjoint from $D$. Since we can extricate $D$ from (isotopic copies of) $\Sigma$ in stages, if we show that each $\left.\xi\right|_{M \backslash \Sigma_{i}}$ is tight, this implies the tightness of $\left.\xi\right|_{M}$. Now, for universal tightness, we pass to a large finite cover $\widetilde{M}$ of $M$ and extricate some lift $\widetilde{D}$ of $D$ from the preimage $\widetilde{\Sigma}$ of $\Sigma$. Lifting to a cover has the following advantages:

1. A bypass which is $\# \Gamma$-increasing can be made trivial by using the residual finiteness of $M$.

2. A bypass whose attaching arc intersects only two distinct curves but is not trivial or $\# \Gamma$-increasing can be made to intersect three distinct curves by Theorem 1.6.

Therefore, by lifting as necessary so that each bypass satisfies Conditions 1 and 2 above, we obtain a sequence

$$
\left(\widetilde{M}_{0}=M, \widetilde{\Sigma}_{0}=\Sigma, \widetilde{\xi}_{0}=\xi\right),\left(\widetilde{M}_{1}, \widetilde{\Sigma}_{1}, \widetilde{\xi}_{1}\right), \ldots,\left(\widetilde{M}_{n}, \widetilde{\Sigma}_{n}, \widetilde{\xi}_{n}\right),
$$

where $\widetilde{\Sigma}_{i} \subset \widetilde{M}_{i}, \widetilde{M}_{i+1}$ is a finite cover of $\widetilde{M}_{i}, \widetilde{\xi}_{i}$ is the pullback of $\xi$ to $\widetilde{M}_{i}, \widetilde{\Sigma}_{i+1}$ is obtained from the preimage of $\widetilde{\Sigma}_{i}$ by isotoping across a bypass $\mathcal{B}_{\alpha_{i}}$ whose arc of attachment $\alpha_{i}$ intersects three distinct dividing curves, and a lift $\widetilde{D}$ of $D$ in $\widetilde{M}_{n}$ is 
disjoint from $\widetilde{\Sigma}_{n}$. Here $i=0, \ldots, n-1$. If we can show that $\left.\widetilde{\xi}_{i}\right|_{\widetilde{M}_{i} \backslash \widetilde{\Sigma}_{i}}$ is universally tight for all $i$, we are done. This involves making sure that at each step the contact structure is fully straddled and satisfies the conditions of the theorem.

To simplify notation, we will suppress the index $i$ and still write $M$ for the finite cover of $M$ and $\Sigma$ for the preimage of $\Sigma$. Start with $\Sigma$ and $M$, and take a finite cover so that the isotopy takes place across a bypass $\mathcal{B}_{\alpha}$ which intersects three distinct dividing curves. Denote the copies of $\Sigma$ in $M \backslash \Sigma$ by $\Sigma^{j}, j=0,1$, and let $\mathbb{S}^{j}$ be the straddling set for $\Sigma^{j}$. By Proposition $1.3, \Sigma$ is fully straddled on both sides (i.e., on $\Sigma^{0}$ and on $\Sigma^{1}$ ). Suppose we are digging $\mathcal{B}_{\alpha}$ from the $\Sigma^{0}$ side and reattaching $\mathcal{B}_{\alpha}$ onto the $\Sigma^{1}$ side. This gives us the surface $\Sigma^{\prime}$, which is parallel to and disjoint from $\Sigma$. By Proposition 1.5, the curve $\gamma$ straddled by $\alpha$ must be in $\Gamma\left(\mathbb{S}^{0}\right)$, and hence is straddled by an arc $\beta_{0}$ of $\mathbb{S}^{0}$. Since $\gamma$ is straddled by an element of $\mathbb{S}^{0}$ if and only if $\gamma$ is not straddled by any element of $\mathbb{S}^{1}$, it follows that the parallel curve $\gamma^{\prime}$ is straddled by an $\operatorname{arc} \beta_{1} \in \mathbb{S}^{1}$.

As in the proof of Proposition 1.5, we may use Theorem 1.6 and pass to a larger finite cover so that $\alpha$ and $\beta_{0}$ are both disjoint from $\beta_{1}$.

It is clear that digging $\mathcal{B}_{\alpha}$ preserves universal tightness. Since $\alpha$ and $\beta_{1}$ are disjoint, Proposition 1.1 tells us that, on $M \backslash \Sigma$, digging $\mathcal{B}_{\alpha}$ is equivalent to attaching a bypass $\mathcal{B}_{\beta_{1}}^{\prime}$ along $\beta_{1}$ onto the $\Sigma^{0}$ side, i.e., $M \backslash\left(\Sigma \cup \mathcal{B}_{\alpha}\right)$ is contactomorphic to $M^{\prime}=\overline{(M \backslash \Sigma) \cup N\left(\mathcal{B}_{\beta_{1}}^{\prime}\right)}$, where $N(F)$ is a small neighborhood of $F$. Applying Proposition 1.1 once more, attaching a bypass $\mathcal{B}_{\beta_{1}}^{\prime}$ along $\beta_{1}$ onto the $\Sigma^{0}$ side is equivalent to digging $\mathcal{B}_{\beta_{0}}$. If we write $\partial M^{\prime}=\left(\Sigma^{\prime}\right)^{0} \sqcup \Sigma^{1}$, then $\left(\Sigma^{\prime}\right)^{0} \subset M^{\prime}$ is fully straddled, with a straddling set obtained by dropping $\beta_{0}$ from $\mathbb{S}^{0}$. By yet another application of Proposition 1.1, attaching a bypass $\mathcal{B}_{\alpha}^{\prime}$ to $\Sigma^{1} \subset M^{\prime}$ along $\alpha$ is equivalent to digging a bypass $\mathcal{B}_{\beta_{1}}$ along $\beta_{1}$. In other words, $M^{\prime} \cup N\left(\mathcal{B}_{\alpha}^{\prime}\right)$ is contactomorphic to $M^{\prime} \backslash \mathcal{B}_{\beta_{1}}$ and also to $M \backslash \Sigma^{\prime}$. Therefore, $\left.\xi\right|_{M \backslash \Sigma^{\prime}}$ is universally tight and is fully straddled by $\mathbb{S}^{0}-\left\{\beta_{0}\right\}$ on the $\left(\Sigma^{\prime}\right)^{0}$ side and $\mathbb{S}^{1}-\left\{\beta_{1}\right\}$ on the $\left(\Sigma^{\prime}\right)^{1}$ side (by applying Proposition 1.4).

We can now inductively construct $\left(\widetilde{M}_{i}, \widetilde{\Sigma}_{i}, \widetilde{\xi}_{i}\right)$ that are fully straddled on both sides which satisfy the conditions of the theorem, by choosing finite covers where Conditions 1 and 2 are met. This ensures universal tightness of each step and finishes the proof.

\section{The Gabai-Eliashberg-Thurston Theorem}

Proof of Theorem 0.1. According to Gabai [10], there is a well-groomed sutured manifold decomposition of $M$,

$$
M \stackrel{\Sigma}{\stackrel{\Sigma}{\rightsquigarrow}}\left(M_{1}, \gamma_{1}\right) \stackrel{S_{1}}{\rightsquigarrow} \ldots \stackrel{S_{n-1}}{\rightsquigarrow}\left(M_{n}, \gamma_{n}\right)=\cup\left(B^{3}, S^{1} \times I\right),
$$

where $\Sigma$ is an oriented nonseparating surface representing a nontrivial class in $H_{2}(M ; \mathbb{Z})$. Since the toroidal case was already discussed in [20], we assume that 
$M$ is atoroidal and the genus of $\Sigma$ is $\geq 2$. Since $M$ and $\Sigma$ are closed, $\gamma_{1}=\emptyset$. For $i \geq 1, S_{i}$ may be chosen to have nonempty boundary (see [20], Theorem 1.3 for a statement of this version of Gabai's theorem). It follows that for $i \geq 2,\left(M_{i}, \gamma_{i}\right)$ has annular sutures, that is, all sutures are annuli, every component of $\partial M_{i}$ contains at least one suture, and every component of $M_{i}$ has nonempty boundary.

By the results of [20], the sutured manifold decomposition

$$
\left(M_{2}, \gamma_{2}\right) \stackrel{S_{2}}{\rightsquigarrow}\left(M_{3}, \gamma_{3}\right) \stackrel{S_{3}}{\rightsquigarrow} \ldots \stackrel{S_{n-1}}{\rightsquigarrow}\left(M_{n}, \gamma_{n}\right)=\cup\left(B^{3}, S^{1} \times I\right),
$$

gives rise to a convex decomposition

$$
\left(M_{2}, \Gamma_{2}\right) \stackrel{\left(S_{2}^{\prime}, \sigma_{2}\right)}{\sim}\left(M_{3}, \Gamma_{3}\right) \stackrel{\left(S_{3}^{\prime}, \sigma_{3}\right)}{\sim} \ldots \stackrel{\left(S_{n-1}^{\prime}, \sigma_{n-1}\right)}{\rightsquigarrow}\left(M_{n}, \Gamma_{n}\right)=\cup\left(B^{3}, S^{1}\right),
$$

and then (and this requires that each of the $\left(M_{i}, \Gamma_{i}\right)$ above have annular sutures) $\left(M_{2}, \Gamma_{2}\right)$ carries a universally tight contact structure by Theorem 6.1 of [19]. Note that the proof of Theorem 6.1 in [19] uses the perturbation of a taut foliation into a universally tight contact structure; we gave a foliation-theory-free proof of the same fact in $[20]$.

Gabai's construction gives $\left(M_{1}, \gamma_{1}=\emptyset\right) \stackrel{S_{1}}{\rightsquigarrow}\left(M_{2}, \gamma_{2}\right)$, so to apply Theorem 6.1 of [19], it is necessary to produce a convex structure $\left(M_{1}, \Gamma_{1}\right)$ with annular sutures (in particular, $\left.\Gamma_{1} \neq \emptyset\right)$ and a splitting surface $\left(S_{1}^{\prime}, \sigma_{1}\right)$ such that $\left(M_{1}, \Gamma_{1}\right) \stackrel{\substack{S_{1}^{\prime}, \sigma_{1} \\ \rightsquigarrow}}{\rightsquigarrow}$ $\left(M_{2}, \Gamma_{2}\right)$. Let $\Sigma^{0}$ and $\Sigma^{1}$ be the components of $\partial M_{1}$ corresponding to the original splitting surface $\Sigma$. Since $S_{1}$ is well-groomed, the components of $S_{1} \cap \Sigma^{i}, i=0,1$, are parallel oriented nonseparating curves in the isotopy class $s_{i}$. Let $A_{i}$ be an annular neighborhood of a curve dual to $s_{i}$, and denote $\partial A_{i}=\delta_{i} \sqcup \delta_{i}^{\prime}$. (Here, two closed curves $\alpha, \beta$ on the same closed surface are dual if $|\alpha \cap \beta|=1$.) Now define $\Gamma_{1}=\delta_{0} \sqcup \delta_{0}^{\prime} \sqcup \delta_{1} \sqcup \delta_{1}^{\prime}$. The convex structure $\left(M_{1}, \Gamma_{1}\right)$ is defined by decreeing that $\Sigma^{i} \backslash A_{i} \subset\left(\Sigma^{i}\right)_{+}$and $A_{i} \subset\left(\Sigma^{i}\right)_{-}$if and only if the orientation induced from $\Sigma$ agrees with the outward-pointing normal orientation on $\Sigma^{i}$.

Now, $\left(S_{1}^{\prime}, \sigma_{1}\right)$ is defined so that $S_{1}^{\prime}=S_{1}$ and $\sigma_{1}$ is the unique dividing set which is $\partial$-parallel and gives rise to $\left(M_{2}, \Gamma_{2}\right)$ after the splitting. The bypasses corresponding to the $\partial$-parallel dividing curves straddle curves of $\Gamma_{1}$. Due to the well-grooming of $S_{1}$, there is a unique choice of straddled curve for each pair $\delta_{i} \sqcup \delta_{i}^{\prime}$. It now follows that there is a universally tight contact structure on $\left(M_{1}, \Gamma_{1}\right)$ and that $\partial M_{1}$ is fully straddled with $\Gamma(\mathbb{S})=\delta_{0} \sqcup \delta_{1}$, for example.

Next consider $M=M_{1} \cup(\Sigma \times[0,1])$, where we identify $\Sigma^{i}$ with $\Sigma \times\{i\}, i=0,1$. By Theorem 0.5, there is a (unique) universally tight contact structure on $\Sigma \times I$ with $\Gamma_{\partial(\Sigma \times I)}=\delta_{0} \sqcup \delta_{0}^{\prime} \sqcup \delta_{1} \sqcup \delta_{1}^{\prime}$ and $\Gamma(\mathbb{S})=\delta_{0}^{\prime} \sqcup \delta_{1}^{\prime}$. We may then apply Theorem 0.4 twice to obtain a universally tight contact structure on $M$. 


\section{Appendix}

In this section we show that the 4 universally tight contact structures described in Theorem 0.5 exist. On $\Sigma \times[0,1]$, we have boundary conditions $\Gamma_{\Sigma_{i}}=\gamma_{i} \sqcup \gamma_{i}^{\prime}$, $i=0,1$. For convenience, we introduce notation which singles out the straddled curve on each $\Sigma_{i}$ - the 4 potentially tight contact structures, distinguished by the 4 possible choices of the set of straddled curves $\Gamma(\mathbb{S})$, are written $\left[\gamma_{0}, \gamma_{1}\right],\left[\gamma_{0}, \gamma_{1}^{\prime}\right]$, $\left[\gamma_{0}^{\prime}, \gamma_{1}\right]$, and $\left[\gamma_{0}^{\prime}, \gamma_{1}^{\prime}\right]$. The next proposition shows how to construct two of these structures in a special case.

Proposition 3.1. Assume curves $\gamma_{0}$ and $\gamma_{1}$ on $\Sigma$ have a common dual curve $\delta$. For each $i=0,1$, orient $\delta$ and then orient $\gamma_{i}$ and $\gamma_{i}^{\prime}$ using the boundary orientation $\partial\left(\Sigma_{i}\right)_{-}=\gamma_{i}+\gamma_{i}^{\prime}$ and the requirement that $\left\langle\gamma_{i}, \delta\right\rangle=+1$. (Here $\langle$,$\rangle is the$ intersection form.) Then two of the universally tight contact structures described in Theorem 0.5 exist and are written as $\left[\gamma_{0}, \gamma_{1}\right]$ and $\left[\gamma_{0}^{\prime}, \gamma_{1}^{\prime}\right]$.

Proof. The desired contact structures are built by specifying a convex decomposition of $\left(\Sigma \times I, \Gamma_{\Sigma_{0}} \sqcup \Gamma_{\Sigma_{1}}\right)$. Let $\delta \times I$ be the first splitting surface, and let $\Gamma_{\delta \times I}$ consist of two $\partial$-parallel dividing curves. Figure $2(\mathrm{~A})$ shows $(\Sigma \times I) \backslash(\delta \times I)$. Figure 2(B) shows one possible dividing curve configuration on $\Sigma \times\{0\}$. After rounding corners and isotoping the dividing set (Figure $2(\mathrm{C})$ ), we obtain a convex structure on $M_{1}=(\Sigma \times I) \backslash(\delta \times I)$ where $\Gamma_{M_{1}}$ consists of a pair of dividing curves, one at the core of each vertical annulus on $\partial M_{1}$.

There is now a canonical "product" decomposition of $\left(M_{1}, \Gamma_{M_{1}}\right)$ along disks which intersect the dividing curves twice. We show just one step of the remaining decomposition. Let $\varepsilon$ be an arc in $\Sigma \backslash \delta$ that connects the two vertical annuli of $\partial M_{1}$. Figure 2(D) shows the unique convex structure on $\varepsilon \times I$ that is consistent with the intersection pattern of $\varepsilon \times I$ and $\Gamma_{M_{1}}$. Splitting along $\varepsilon \times I$, rounding corners, and isotoping produces $\left(M_{2}, \Gamma_{M_{2}}\right)$ and is shown in Figures 2(E) and $(\mathrm{F})$. Continuing in this fashion produces a convex decomposition of $\left(\Sigma \times I, \Gamma_{\Sigma_{0}} \sqcup \Gamma_{\Sigma_{1}}\right)$, where all splitting surfaces are $\partial$-parallel. By Theorem 0.3 , this convex decomposition defines a universally tight contact structure on $\Sigma \times I$.

Depending on how the dividing set on $\delta \times I$ was chosen, the universally tight contact structure just constructed has both $\gamma_{0}$ and $\gamma_{1}$ straddled, or both $\gamma_{0}^{\prime}$ and $\gamma_{1}^{\prime}$ straddled. Therefore, we have constructed both $\left[\gamma_{0}, \gamma_{1}\right]$ and $\left[\gamma_{0}^{\prime}, \gamma_{1}^{\prime}\right]$.

Applying this proposition to the special case of $\gamma_{0}=\gamma_{1}$ immediately gives:

Corollary 3.2. If $\gamma$ is a nonseparating curve in $\Sigma$, then there exist universally tight contact structures of the form $[\gamma, \gamma]$ and $\left[\gamma^{\prime}, \gamma^{\prime}\right]$.

The rest of the proof consists of small successive improvements of Proposition 3.1, using the Gluing Theorem. Recall that the Gluing Theorem implies that two universally tight contact structures on $\Sigma \times I$ with $\Gamma_{\Sigma_{i}}$ consisting of two parallel 
(A)

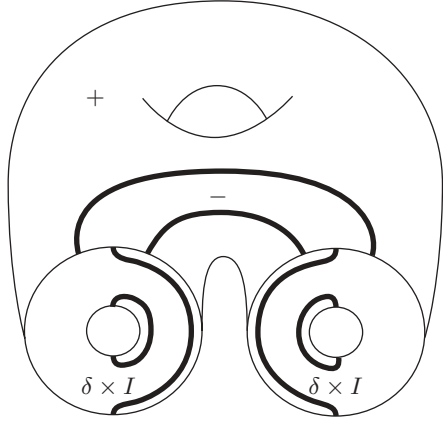

(B)

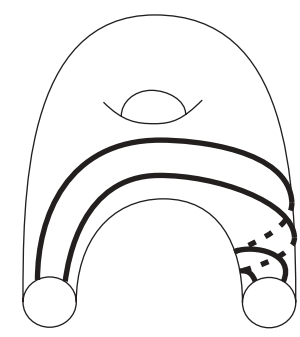

$(\Sigma \backslash \delta) \times\{0\}$

(C)
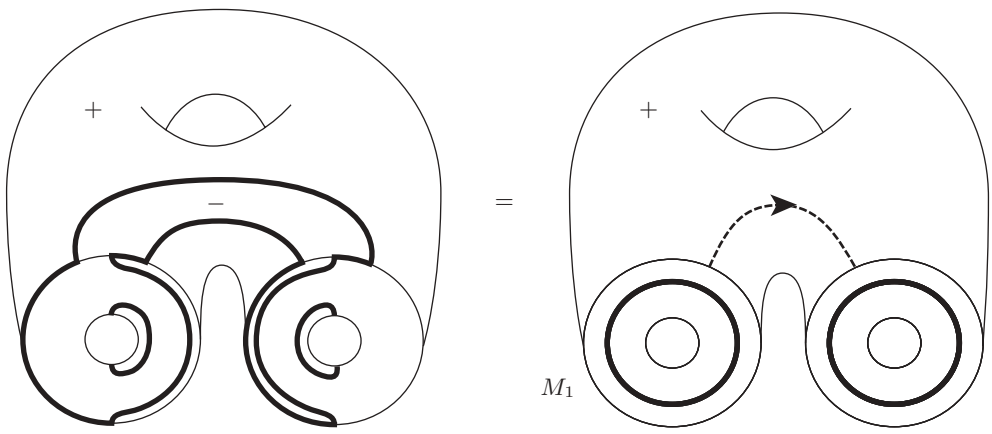

(D)

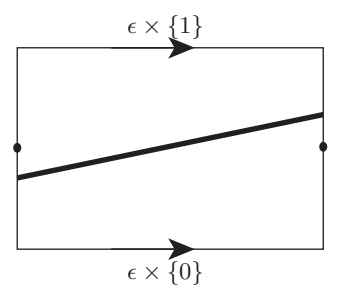

(E)

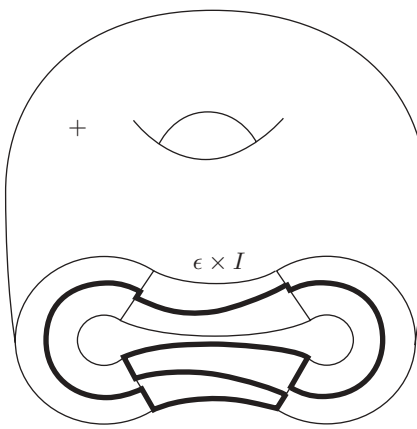

(F)

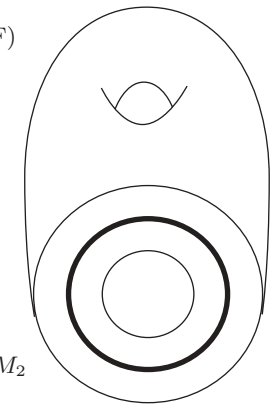

FIG. 2

curves can be glued across a common boundary component to give a universally tight contact structure if and only if the dividing sets match, and the curve that is straddled on one side is not straddled on the other. An application of the Gluing Theorem then gives:

Proposition 3.3. If $\gamma$ is a nonseparating curve in $\Sigma$, then the 4 universally tight contact structures $[\gamma, \gamma],\left[\gamma, \gamma^{\prime}\right],\left[\gamma^{\prime}, \gamma\right]$, and $\left[\gamma^{\prime}, \gamma\right]$ on $\Sigma \times I$ exist. 
Proof. By Corollary 3.2, we know that universally tight contact structures of the form $[\gamma, \gamma]$ and $\left[\gamma^{\prime}, \gamma^{\prime}\right]$ exist. The Gluing Theorem implies the tightness of $\left[\gamma, \gamma^{\prime}\right]=$ $[\gamma, \gamma] \cup\left[\gamma^{\prime}, \gamma^{\prime}\right]$ and $\left[\gamma^{\prime}, \gamma\right]=\left[\gamma^{\prime}, \gamma^{\prime}\right] \cup[\gamma, \gamma]$.

This can be used to strengthen Proposition 3.1.

Proposition 3.4. If $\gamma_{0}$ and $\gamma_{1}$ on $\Sigma$ have a common dual curve $\delta$, then the 4 universally tight contact structures $\left[\gamma_{0}, \gamma_{1}\right],\left[\gamma_{0}, \gamma_{1}^{\prime}\right],\left[\gamma_{0}^{\prime}, \gamma_{1}\right]$, and $\left[\gamma_{0}^{\prime}, \gamma_{1}^{\prime}\right]$ exist.

Proof. Again, the Gluing Theorem implies the universal tightness of $\left[\gamma_{0}, \gamma_{1}^{\prime}\right]=$ $\left[\gamma_{0}, \gamma_{1}\right] \cup\left[\gamma_{1}^{\prime}, \gamma_{1}^{\prime}\right]$ and $\left[\gamma_{0}^{\prime}, \gamma_{1}\right]=\left[\gamma_{0}^{\prime}, \gamma_{1}^{\prime}\right] \cup\left[\gamma_{1}, \gamma_{1}\right]$.

The next theorem is the existence portion of Theorem 0.5.

Theorem 3.5. Let $\gamma_{i} \subset \Sigma, i=0,1$, be nonseparating curves, and let $\gamma_{i}^{\prime}$ be their parallel copies. Then there exist tight contact structures of all 4 types: $\left[\gamma_{0}, \gamma_{1}\right],\left[\gamma_{0}, \gamma_{1}^{\prime}\right],\left[\gamma_{0}^{\prime}, \gamma_{1}\right]$, and $\left[\gamma_{0}^{\prime}, \gamma_{1}^{\prime}\right]$.

Proof. Let $\gamma_{0}=\beta_{0}, \beta_{1}, \beta_{2}, \ldots, \beta_{n}=\gamma_{1}$ be a sequence of nonseparating curves such that $\left|\beta_{i} \cap \beta_{i+1}\right|=1$. Since adjacent curves $\beta_{i}, \beta_{i+1}$ have common duals, we can construct a universally tight contact structure by stacking a sequence of universally tight contact structures supplied by Proposition 3.4. As long as we ensure that the same curve is not straddled on both sides of the gluing surface, the resulting contact structure will be universally tight. It is clear, with a final application of Proposition 3.3 to adjust the straddled curves at the ends, that all 4 configurations of straddled curves can be achieved.

\section{References}

[1] E. Allman and E. Hamilton, Abelian subgroups of finitely generated Kleinian groups are separable, Bull. London Math. Soc. 31 (1999), 163-172.

[2] D. Bennequin, Entrelacements et équations de Pfaff, Astérisque 107-108 (1983), 87-161.

[3] V. Colin, Chirurgies d'indice un et isotopies de sphères dans les variétés de contact tendues, C. R. Acad. Sci. Paris Sér. I Math. 324 (1997), 659-663.

[4] V. Colin, Recollement de variétés de contact tendues, Bull. Soc. Math. France 127 (1999), 43-69.

[5] Y. Eliashberg, Classification of overtwisted contact structures on 3-manifolds, Invent. Math. 98 (1989), 623-637.

[6] Y. Eliashberg, Topological characterization of Stein manifolds of dimension $>2$, Internat. J. Math. 1 (1990), 29-46.

[7] Y. Eliashberg, Filling by holomorphic discs and its applications, Geometry of lowdimensional manifolds, 2 (Durham, 1989), 45-67, London Math. Soc. Lecture Note Ser. 151, Cambridge Univ. Press, Cambridge, 1990.

[8] Y. Eliashberg, Contact 3-manifolds twenty years since J. Martinet's work, Ann. Inst. Fourier (Grenoble) 42 (1992), 165-192. 
[9] Y. Eliashberg and W. Thurston, Confoliations, University Lecture Series 13, Amer. Math. Soc., Providence, RI, 1998.

[10] D. Gabai, Foliations and the topology of 3-manifolds, J. Differential Geom. 18 (1983), 445-503.

[11] E. Giroux, Convexité en topologie de contact, Comment. Math. Helv. 66 (1991), 637-677.

[12] E. Giroux, Une infinité de structures de contact tendues sur une infinité de variétés, Invent. Math. 135 (1999), 789-802.

[13] E. Giroux, Structures de contact en dimension trois et bifurcations des feuilletages de surfaces, Invent. Math. 141 (2000), 615-689.

[14] R. Gompf, Handlebody construction of Stein surfaces, Ann. of Math. (2) 148 (1998), 619-693.

[15] M. Gromov, Pseudo-holomorphic curves in symplectic manifolds, Invent. Math. 82 (1985), $307-347$.

[16] K. Honda, On the classification of tight contact structures I, Geom. Topol. 4 (2000), 309368 (electronic).

[17] K. Honda, On the classification of tight contact structures II, J. Differential Geom. 55 (2000), 83-143.

[18] K. Honda, Gluing tight contact structures, Duke Math. J. 115 (2002), 435-478.

[19] K. Honda, W. Kazez and G. Matić, Tight contact structures and taut foliations, Geom. Topol. 4 (2000), 219-242.

[20] K. Honda, W. Kazez and G. Matić, Convex decomposition theory, Internat. Math. Res. Notices 2002, 55-88.

[21] K. Honda, W. Kazez and G. Matić, Tight contact structures on fibered hyperbolic 3manifolds, J. Differential Geom. 64 (2003), 305-358.

[22] Y. Kanda, The classification of tight contact structures on the 3-torus, Comm. Anal. Geom. 5 (1997), 413-438.

[23] Y. Kanda, On the Thurston-Bennequin invariant of Legendrian knots and non exactness of Bennequin's inequality, Invent. Math. 133 (1998), 227-242.

Ko Honda

University of Southern California

Los Angeles

CA 90089

USA

e-mail: khonda@math.usc.edu url: http://math.usc.edu/ ${ }^{\sim}$ khonda

Gordana Matić

University of Georgia

Athens

GA 30602

USA

e-mail: gordana@math.uga.edu

url: http://www.math.uga.edu/ ${ }^{\sim}$ gordana

(Received: November 12, 2002)
William H. Kazez

University of Georgia

Athens

GA 30602

USA

e-mail: will@math.uga.edu url: http://www.math.uga.edu/ ${ }^{\sim}$ will 